\title{
Example-Based Fractured Appearance
}

\author{
L. Glondu ${ }^{1}$, L. Muguercia ${ }^{2}$, M. Marchal ${ }^{1}$, C. Bosch $^{2}$, H. Rushmeier ${ }^{3}$, G. Dumont ${ }^{1}$ and G. Drettakis ${ }^{4}$ \\ ${ }^{1}$ INRIA / ENS Cachan / INSA Rennes, France \\ ${ }^{2}$ ViRVIG - Universitat de Girona, Spain \\ ${ }^{3}$ Yale University, USA \\ ${ }^{4}$ REVES / INRIA Sophia-Antipolis, France
}
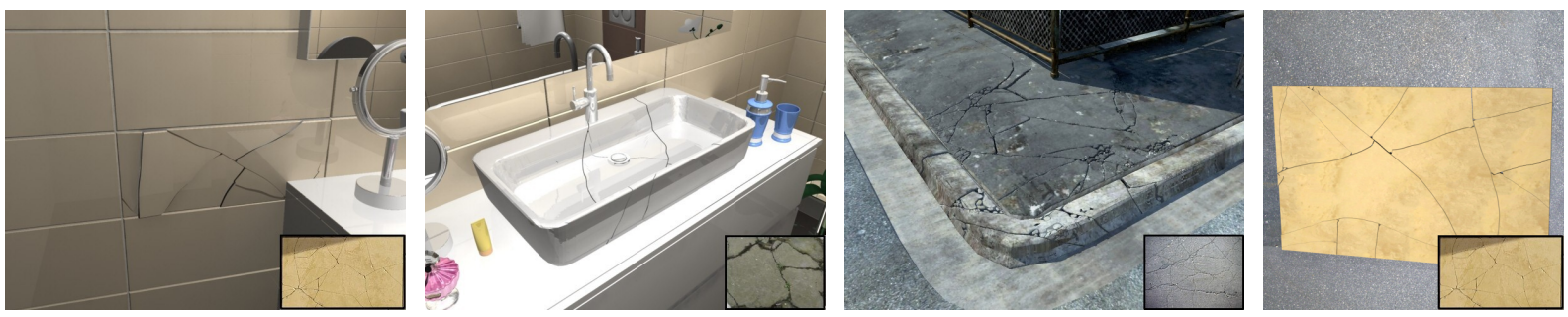

Figure 1: Application of our example-based fracturing method on different scenes. Photographs of input fracture patterns are shown in the insets. Left to right: (1) a bathroom tile has been broken, and each fragment can be manipulated separately. (2) Fracture pattern obtained from a ground tile photograph applied on a basin. (3) Sidewalk and curb from a urban scene fractured using the same pattern. (4) A similar but not identical cracked tile created from a photographic exemplar.

\begin{abstract}
A common weathering effect is the appearance of cracks due to material fractures. Previous exemplar-based aging and weathering methods have either reused images or sought to replicate observed patterns exactly. We introduce a new approach to exemplar-based modeling that creates weathered patterns on synthetic objects by matching the statistics of fracture patterns in a photograph. We present a user study to determine which statistics are correlated to visual similarity and how they are perceived by the user. We then describe a revised physically-based fracture model capable of producing a wide range of crack patterns at interactive rates. We demonstrate how a Bayesian optimization method can determine the parameters of this model so it can produce a pattern with the same key statistics as an exemplar. Finally, we present results using our approach and various exemplars to produce a variety of fracture effects in synthetic renderings of complex environments. The speed of the fracture simulation allows interactive previews of the fractured results and its application on large scale environments.
\end{abstract}

Categories and Subject Descriptors (according to ACM CCS): I.3.7 [Computer Graphics]: Three-Dimensional Graphics and Realism—Color, shading, shadowing, and texture I.6.3 [Simulation and Modeling]: Applications-

\section{Introduction}

Cracks and fractures are important visual effects which we encounter in everyday life. Pavements and roads suffer degradation over time, and often have many cracks, sometimes resulting in fragments being detached. Similarly, tiles are often sensitive to a strong impact and are frequently fractured. Various simulation methods exist that can reproduce the physical fracture process e.g., [OBH02, IO06, MG04]. These methods are controlled by a set of physical parameters and the choice of these parameters determines the visual appearance of the degraded surface. It is however hard to find the correct values to achieve a desired visual effect.

The goal of this paper is to extract fracture simulation parameters from a photograph of cracks with a specific visual appearance and rapidly allow application of similar fracture patterns on other objects. To do this, we first need a way to determine whether two fracture patterns are similar, and a method to reproduce this similarity via fracture simulation.

As opposed to recent approaches on example-based simulation [BLR*11], we propose a new approach that estimates 
parameters by matching statistics rather than matching exact patterns. We perform a user study to determine which statistics form a valid metric for visual similarity. We use the metric in an optimization to find simulation parameters. This approach thus facilitates the generation of similar looking, but not identical, fractures.

Our method starts with an input photograph of a given crack pattern. We extract statistics of the fractures, using features identified in [SDF*10], such as fragment area, crack edge length, orientation or straightness. We then perform a perceptual study to establish that these statistics are indeed correlated with visual similarity between fracture patterns, and to determine the relative importance of each statistic in the perception of similarity. The statistics and the results of the study allow us to define a metric for similarity of fracture patterns. We extend existing fracture simulation techniques so we can optimize their parameters and generate a large variety of patterns found in photographs of fractures. We then introduce an optimization approach to match the statistics of the input photograph, resulting in a set of parameters reproducing similar looking patterns on different surfaces. Finally, we develop a fracture modeling interface which allows interactive addition of fractures to a model of a city neighborhood, based on example photographs.

In summary, our contributions are:

- A perceptual study to establish the correlation of fracture statistics to visual similarity of fracture patterns. This gives a metric measuring fracture pattern similarity based on statistics.

- An optimization approach based on the similarity metric that extracts simulation parameters, allowing interactive generation of fractures with appearance similar to the example. To allow this optimization, we extend previous physically-based fracture simulation approaches.

The speed of our approach makes our method applicable to large-scale models, and provides an interactive fracture modeling interface to apply fractures to various models based on examples from photographs. We illustrate this process on different scenes e.g., a city neighborhood (Fig. 7) or interior scenes (Fig. 1). By using a true volumetric simulation, we can simulate changes in the geometry due to the weathering effect, as well as surface patterns. In the results section, we show several examples of photographs of fracture patterns and the corresponding simulations resulting from the optimization process.

\section{Related Work}

Aging and Weathering Simulating aging and weathering is of special relevance when modeling realistic environments. Previous work has focused on physically-based and phenomenological simulations to reproduce these effects [DPH96, MG08]. However, choosing appropriate simulation parameters to match a specific effect becomes a te- dious task. Recent approaches moved to data-driven techniques to directly transfer weathering effects from photographs [WTL* $\left.06, G^{*} R^{*} 06\right]$. These techniques simplify the modeling process, but are restricted by the kind of information available in the examples. The combination of these two approaches has been recently introduced for simulating flow stains, where the parameters of the simulation are fitted from an input example [BLR* $\left.{ }^{*} 11\right]$. This method offers several advantages, such as avoiding the selection of parameters while still providing enough versatility. However, applying the same idea to other weathering phenomena is not straightforward. Major issues include selecting a suitable simulation procedure and defining a metric for "matching" the photograph of a desired effect. The simulation must be fast, and capable of generating the variety of structures observed. Since a process such as fracture is difficult to control, we need an approach that attempts something other than producing the identical weathered pattern.

Fracture Simulation Fracture simulation is now commonplace in the computer graphics community. In [TF88], the first attempts of physically-based fracture from a finite difference simulation were presented. Later, O'Brien and Hodgins [OH99] introduced rich simulations of brittle fracture from conventional Finite Element Method simulation. Their work was also extended to ductile fracture [OBH02]. Quasistatic analysis for impact-based fracture has also been studied [MMDJ01, MG04, BHTF07, ZJ10].

Fracture initiation and propagation are the two steps of a fracture simulation. For fracture initiation, Iben and O'Brien [IO06] simulate surface crack patterns using a heuristically specified stress field. This field evolves over time on a triangular mesh, opening cracks at locations of highest stress. However, their method relies on a specified stress field and can not take into account the deformations of the body due to loading forces. To model different loading forces in an interactive way, a fracture model needs to handle volumetric meshes. Fracture propagation can be computed on the physical meshes or only on the surface such as the method presented in [GMD12], where the authors use an implicit surface that propagates over the volumetric mesh of the simulated body to sample the fracture geometry. This method handles crack intersections in a robust and efficient way. In its original version it cannot match all the classes of fracture statistics we observed. We introduce a stress relaxation component to the method of [GMD12] that reproduces a larger variety of patterns observed in photographs.

Besides simulation, crack generation has also been reproduced using procedural approaches [Mou05, LM09]. In [Mou05], the author proposes an image filter that transforms an input line drawing into an image of a fractured surface. Hsien et al. [HT06] render crack patterns and animate their propagation on 3D object surfaces, extracting patterns by image processing operations from vectorized crack images. Desbenoit et al. [DGA05] create a library of crack 
patterns from real-world images as we do, but these are directly applied onto new objects without relying on a physical simulation nor on a parameter estimation. All these approaches typically reproduce a specific pattern, lacking flexibility or a physical basis of fracture formation. [GC01] describe a semi-physical method for modeling the propagation of cracks on a 3D surface based on a multi-layer Cellular Automaton, giving more control to the user. Our approach can be seen as combining the best of simulation and procedural approaches, since we extract simulation parameters from photographs. Our statistical approach to simulation allows us to generate varieties of physically plausible patterns which are visually similar.

Statistical Models for Fractures The statistical properties of fracture patterns have been analyzed in fields such as mechanics [Gri21], paleontology [CBRY09] and other fields [Clo55]. Their main goal is to use statistics to understand fractured objects based on shapes and adjacency information. In Computer Graphics, Valette et al. [VPLL08] estimated densities of area, length and connectivity of crack patterns to validate their simulation results against real patterns. Shin et al. [SDF* 10$]$ recently analyzed fracture patterns observed in wall paintings, in order to improve the matching of fresco fragments [FSTF*11]. We rely on a similar set of statistics, but we use them to fit simulation parameters rather than reconstructing the fractured object. We also study the perceptual effect of these statistics on visual similarity.

\section{Overview}

Starting with a photograph of the desired pattern, the location of the cracks is first specified by the user through a standard graphics editor. The obtained pattern is then processed into edges, junctions and fragments, and a set of statistical properties are obtained from them similar to [SDF* 10]. The various statistics extracted have different levels of influence on the resulting visual similarity to the exemplar. To investigate this effect we performed a user study to determine whether these statistics are correlated to visual similarity and the relative importance of the different statistics. The results of the perceptual study allow us to choose the appropriate statistics to use for our optimization.

Our main goal is to fit parameters of a fracture simulation using the extracted statistics. This is achieved through a Bayesian optimization process, which refines the parameters trying to match the statistics between the simulated and input models. We notably introduce a new fracture similarity metric based on the statistics previously evaluated in the user study. We use a real-time fracture model specially tailored for this, combining both physically-based properties with parameters that are effectively fitted within this process.

Once the simulation parameters have been estimated, we generate similar patterns on new scenes. We propose an interactive modeller where fracture patterns can easily be created, edited and finally propagated into similar objects, which facilitates the modeling task on large scale scenes.

\section{Statistical Pattern Similarity}

Our approach requires the determination of the parameters of a simulation that will produce a crack pattern that is visually similar but not identical to the exemplar image. Our hypothesis is that patterns that appear similar can be identified by comparing statistics of crack patterns. We needed to perform a study to confirm that the statistics of the patterns are related to similarity of appearance, and to determine which statistics are most important.

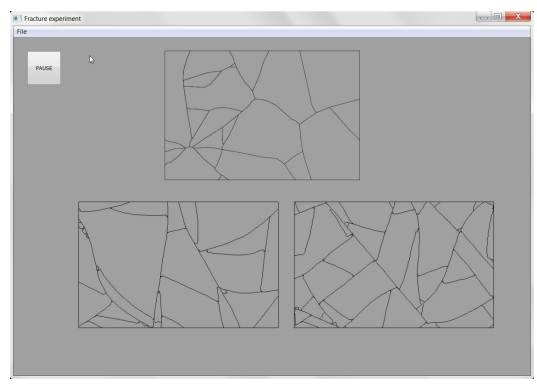

Figure 2: The interface for the user study. Above we show the reference fracture pattern taken from an input photograph. Below, two patterns for which different statistics have been matched (Left: optimization matched the three statistics, Right: the optimization matched the crack length distribution). The user is asked to select the most visually similar pattern to the reference.

We designed a user study to determine the relative importance of the various statistics involved. We gathered a list of potential statistics from previous work [SDF $\left.{ }^{*} 10\right]$ and eliminated redundant measures. Three types of statistics were finally considered: fragment, crack and junction statistics, which we refer to as type $S 1, S 2$ and $S 3$. Fragment statistics are the number and area of the fragments $(S 1)$, crack statistics measure the length and number of cracks $(S 2)$, while junction statistics are the density of junctions (S3).

We performed a 2-alternative forced choice experiment. The interface of the user study can be seen in Fig. 2. At the top is an image of a reference pattern manually extracted from a photograph. Each lower image is generated to match the top image for a subset of the statistics (e.g., S1 or $\mathrm{S} 2+\mathrm{S} 3$ ). We used brute force optimization to determine parameters of the simulation. The users then had to compare the lower two images and choose the one that looked most visually similar to the real example. We used a "linedrawing" rendering style to avoid the use of other cues such as texture.

Experimental Apparatus The user was instructed to select their preferred fracture pattern in terms of similarity to the 


\begin{tabular}{|r|c|c|c|c|c|c|c|}
\hline & $S 1$ & $S 2$ & $S 3$ & $S 1+S 2$ & $S 2+S 3$ & $S 1+S 3$ & $S 1+S 2+S 3$ \\
\hline$S 1$ & & 91 & 79.5 & 76 & 72.5 & 50 & 79 \\
$S 2$ & 9 & & 27 & 24.5 & 32 & 22 & 24.5 \\
$S 3$ & 20.5 & 73 & & 42 & 38.5 & 27.5 & 40.5 \\
$S 2+S 3$ & 24 & 75.5 & 58 & & 44.5 & 39.5 & 41 \\
$S 1+S 3$ & 50 & 78 & 61.5 & 55.5 & & 29 & 47.5 \\
$S 1+S 2+S 3$ & 21 & 75.5 & 72.5 & 60.5 & 71 & & 75.5 \\
\hline
\end{tabular}

Table 1: Percentage of instances that the statistic type for the row was chosen over the type for the column. For example, in the first line, patterns with statistic type 11 were selected in 91 percent of the instances where they were compared to patterns with statistic type $S 2$ matched. The light gray values indicate that there was no significant effect found between the two conditions.

real image by clicking on the preferred image. The participants had $7 \mathrm{~s}$ to give their answer. Timeouts or errors were very rare (less than $1 \%$ of the total number of answers) and were ignored in the analysis. The participants had the option to take breaks by pressing a "Pause" button.

Population and Collected Data 20 participants (14 males and 6 females) aged from 23 to 50 (mean=31.82, standard deviation=7.67) performed the experiment. For each pair of conditions, we recorded the choices of the participants and their time to answer.

Experimental Conditions We used a within-subjects design to evaluate seven different pattern conditions. The seven conditions corresponded to an image simulated by optimizing one of the three types of statistics or a combination of these statistics. The conditions were : (i) $S 1$, (ii) $S 2$, (iii) $S 3$, (iv) $S 1+S 2$, (v) $S 2+S 3$, (vi) $S 1+S 3$, (vii) $S 1+S 2+S 3$. We had 5 reference images which are given in supplementary material. All the possible pairs of the different conditions were tested twice in both orders (i.e., left or right image). For each group of possible combinations, the order between the different pairs was randomized as well as the reference image. The experiment lasted approximately 20 minutes.

Results We analyzed the answers of the participants for the different pattern conditions to determine which conditions gave the highest percentages of answers in terms of similarity between the fracture patterns of the real image and the simulation. Each individual performed 212 comparisons. Under the null hypothesis of equal preference between two conditions, the number of times an individual preferred the first condition follows a binomial distribution with parameters 10 and 1/2. After standardization, such a variable can be approximated by a standard normal random variable. Thus, for each pair of conditions, we tested the presence of a preferred condition using an exact binomial test. The p-values were adjusted with a Bonferroni correction. The percentages of time a condition is chosen are given in Table 1.

The analysis showed that $S 1$ was significantly chosen more than all the other conditions, except the combination $S 1+S 3(p=0.1) . S 2$ and $S 3$ were significantly chosen less than all the other conditions (lines 2 and 3 of Table 1). No significant effect was found between condition $S 1+S 2$ and
$S 2+S 3(p=0.15)$, as well as between condition $S 2+S 3$ and $S 1+S 2+S 3(p=0.55)$. We can conclude that $S 2$ was always less significantly chosen than all the other conditions, except for the conditions where $S 2$ is included. Condition $S 3$ gives the same results as $S 1$ when combined with it.

These results indicate that matching fracture pattern statistics have a significant impact on the visual similarity of the resulting patterns. If pattern statistics were irrelevant, there would have been no significant effect when different pattern statistics were matched. Further, we conclude that fracture area (S1) and junction statistics (S3) appear to have a dominant effect compared to crack length and number (S2) in perceiving visual similarity of fracture patterns.

The user study thus provides a strong indication of the validity of using statistics as a metric for evaluating visual similarity of fracture patterns. It also determines the relative importance of each statistic, which is used to weight the influence of each in the optimization (Sec. 6.2).

\section{Fracture Simulation}

Our goal is to produce fracture patterns that resemble an example photograph. We use a physically-based fracture simulation to generate new patterns, and we attempt to achieve visual similarity by matching appropriate statistics as determined by the user study. Our fracture simulation is composed of two main steps: (1) crack initiation and (2) crack propagation.

We base our approach on previous models, in particular [IO06] and [GMD12]. We extend the crack initiation and propagation approaches so they better fit our optimization method, and so that classical continuum mechanics can be applied. The notation we use is summarized in Table 2.

\subsection{Crack Initiation}

We use the Finite Element Method (FEM) [ZTZ05] to compute the behavior of the object. The analysis begins by discretizing the object into a set $\mathcal{E}$ of tetrahedral elements $e$. Material properties such as density $\rho$, Young's Modulus $E$, Poisson's ratio $v$ are assigned to the object when specifying the material (e.g., concrete, glass, asphalt, etc.) during scene 


\begin{tabular}{|c|c|c|}
\hline Symbol & Description & Role \\
\hline$E$ & Young's modulus & IN \\
$v$ & Poisson's ratio & IN \\
$\rho$ & Density & IN \\
$a g e$ & Duration of the simulation & IN \\
$R_{c_{m}}$ & Mean of resistance to fracture & IN \\
\hline$A_{i}, f_{i}$ & Noise amplitudes \& frequencies & IN \\
\hline$K$ & Stiffness Matrix & INTER \\
$\Lambda$ & Basis (modal analysis) & INTER \\
$\sigma_{e}$ & Stress at element $e$ & INTER \\
$\sigma_{e}^{0}$ & Initial stress of $e$ & INTER \\
$\mathbf{u}$ & Displacement vector & INTER \\
$\mathbf{f}$ & Force vector & INTER \\
$\Delta t$ & Time step & INTER \\
\hline$d \sigma_{e}$ & Stress increase rate of $e$ & OPT \\
$r_{\text {relax }}$ & Radius of relaxation kernel & OPT \\
$v_{\text {relax }}$ & Relaxation rate & OPT \\
$R_{c_{\text {var }}}$ & Variance of resistance to fracture & OPT \\
$l$ & Loading force magnitude & OPT \\
\hline
\end{tabular}

Table 2: Summary of notation used in fracture simulation. The roles are as follows: IN is an Input parameter, INTER is an intermediate value and OPT is a parameter optimized using the extracted statistics. Note that the five first IN parameters are given as part of the material description, while the noise parameters are set as described in Sec. 5.3.

modeling. Using the material properties, the stiffness matrix $K$ is computed for the elements.

To take into account external loading forces $\mathbf{f}$, we compute the static equilibrium of the simulated body w.r.t. f:

$$
K \mathbf{u}=\mathbf{f},
$$

where the unknown $\mathbf{u}$ is the displacement of the degrees of freedom of the body due to the force vector $\mathbf{f}$. From this equilibrium, we compute the initial stress value $\sigma_{e}^{0}$ for each element using a Hookean law of elasticity. To improve the efficiency of the computation of the static equilibrium, we perform a modal analysis on the body in a precomputation step. This gives us a new coordinate system $\Lambda$ that diagonalizes the stiffness matrix $K$ [GMD12], and in which the Eq. (1) can be solved directly. The magnitude $l$ of the loading forces is optimized in our approach since we do not know its value from the reference images. We use an a priori probability distribution in the optimization process to take into account the randomness of the force position. The effect of a loading force on the simulation is illustrated in Fig. 3.

To simulate crack opening, we use a stress map that evolves over time as proposed in [IO06]. This map is initialized at the beginning of the simulation: the internal stress $\sigma_{e}$ of each element $e$ is initialized with $\sigma_{e}^{0}$. At each time step, the stress is updated with a specified stress increase rate $d \sigma_{e}$ that represents the variation of the stress over time. If the maximum principal tensile stress of an element $e$ exceeds its resistance threshold $R_{C_{e}}$, a fracture is opened at the center of

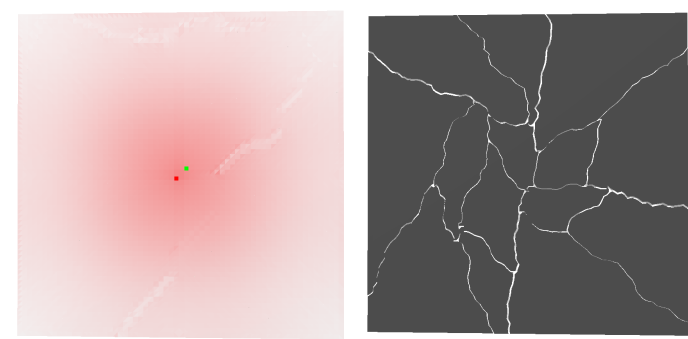

Figure 3: Effect of the loading force on the simulation. A constant loading force is applied from the middle of the tile, leading to higher stress around the middle. Left: stress field computed from the loading force with quasi-static analysis (the intensity of the red value is proportional to the maximum principal stress). Right: A higher concentration of fragments is observed around the middle of the tile.

the element $e$, in a direction orthogonal to the direction of this maximum principal stress. The stress increase rate $d \sigma_{e}$ will be optimized since we do not have any a priori knowledge of the value of this parameter in the exemplar images. The resistance threshold $R_{C_{e}}$ is different in each element, due to the presence of material flaws. The randomness of the parameter is taken into account in the optimization process by the use of an a priori normal distribution with a mean value $R_{c_{m}}$ and a variance $R_{\mathcal{c}_{v a r}}$.

The crack initiation algorithm is summarized below:

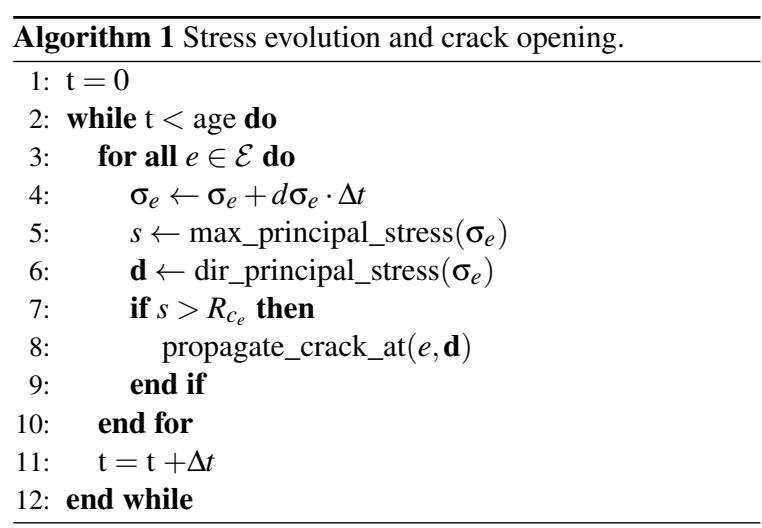

\subsection{Crack Propagation and Stress Relaxation}

We follow the crack propagation method of [GMD12], and we introduce in this paper a stress relaxation computation in order to match more fracture patterns observed in the photographs. In [GMD12], the authors present a fast propagation method where the fracture surface is defined as the intersection of an implicit surface $\mathcal{S}$ and a growing set $\mathcal{E}_{c}$ of elements cut by the fracture surface. Initially, the set of elements cut is composed of one element $e_{0}$, which is the element on which the crack opened. The crack propagates on the neighbors of 
$e_{0}$ that are crossed by the implicit surface $\mathcal{S}$, with an energy criterion to stop the propagation.

When a crack propagates, it alleviates the stress around the crack path. The opening of the crack involves a displacement at the surface that relaxes the surrounding strain and stress. This phenomenon is not modeled in [GMD12]. We extend this method by adding a stress relaxation in order to include the time effects of fracture propagation. Compared to previous work, rather than computing this relaxation by a direct simulation or using e.g., virtual displacements [IO06], we propose a new method that uses an approximate but more efficient simulation of the stress relaxation.

We use a relaxation tensor $\sigma_{\text {relax }}(\mathbf{d})$ in a direction $\mathbf{d}$, computed as $\sigma_{\text {relax }}(\mathbf{d})=\mathbf{d d}^{T} /|\mathbf{d}|$. The relaxation has $|\mathbf{d}|$ as its only non-zero eigenvalue. Subtracting this tensor from a stress tensor $\sigma_{e}$ alleviates it in the direction of $\mathbf{d}$.

The direction $\mathbf{d}$ of the relaxation for an element $e$ is chosen to be the direction orthogonal to both the closest surface normal and the crack propagation direction. Points closer to the crack path will be relaxed more. For efficiency of the simulation, we choose to model this phenomenon using the following polynomial kernel $\phi(d)$, inspired by [MST04]:

$$
\phi(d)=\max \left(0, \frac{1}{r_{\text {relax }}^{6}}\left(r_{\text {relax }}^{2}-d^{2}\right)^{3}\right) .
$$

The kernel is equal to one if the distance $d$ is zero, and zero if $d$ is greater than $r_{\text {relax }}$. Therefore, the value $\sigma_{e_{\text {relaxed }}}$ of the stress of element $e$ after relaxation is computed as:

$$
\sigma_{e_{\text {relaxed }}}=\sigma_{e}-v_{\text {relax }} . \phi(d) \cdot \sigma_{\text {relax }}(\mathbf{d}),
$$

where $d$ is the distance of the center of element $e$ to the fracture surface, and $v_{\text {relax }}$ is a new parameter denoting the stress relaxation rate around the crack. The introduction of the parameters $v_{\text {relax }}$ and $r_{\text {relax }}$ allow finer control of the shapes of the generated fragments, and thus will be determined in the optimization process.

Because we do not want to transform the tensile stress into compressive stress, we check the stress value $s=\mathbf{d}^{T} \sigma_{e_{\text {relaxed }}} \mathbf{d}$ in the direction $\mathbf{d}$. If $s<0$, the relaxation produced compressive stress in the direction $\mathbf{d}$. In this case, we clamp the value to 0 by adding the value $-s * \sigma_{e_{\text {relaxed }}}$ to the stress tensor of the element.

In some patterns, privileged directions are not observed. In that case, we apply a relaxation of the stress in all the directions, and Eq. (3) becomes:

$$
\sigma_{e_{\text {relaxed }}}=\sigma_{e}-v_{\text {relax }} . \phi(d) . I_{d},
$$

where $I_{d}$ is the identity stress tensor that alleviates the stress in all the directions. The effect of the relaxation method on the aspect of the fracture patterns is illustrated in Fig. 4.

To decide whether to use between Eq. (3) or (4), we compute the mean value of the junction angles. If the mean is be-
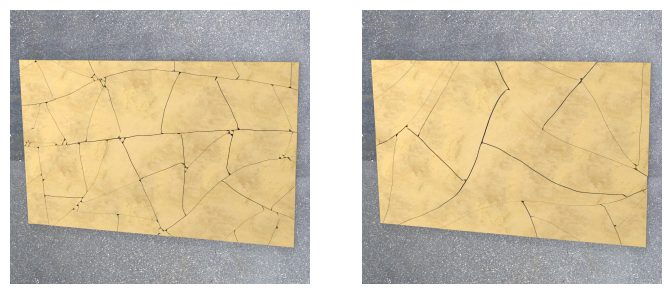

Figure 4: Illustration of the two relaxation methods used. Left: the fracture has a preferred orientation. The stress relaxation of Eq. 3 is used. Right: the pattern does not have a preferred orientation. The stress relaxation of Eq. 4 is used.

tween 75 and 90 degrees (i.e. close to 90 degrees) we choose Eq. (3).

Material anisotropy could be easily added by changing the law of elasticity. However, we observed that the propagation direction of cracks is mainly influenced by other cracks, and these preferred directions are already modeled through our stress relaxation method.

\subsection{Noise}

We use simplex noise [Per02] to reproduce the internal bumpy surfaces that are observed along crack paths (see Fig. 5). This avoids the significant computation cost required to compute the microbranching of cracks. The implicit surface used during the crack propagation is a bumpy plane defined by an origin, a normal vector, and a set of amplitude/frequency couples that define the octave of the noise. The values of the amplitude $A_{i}$ and the frequency $f_{i}$ are chosen manually for each pattern in order to obtain visually similar path variations. We took $i=1 . .4$ for our simulations. In our experience, the choice of these values takes less than one minute for a given 1-D input pattern.

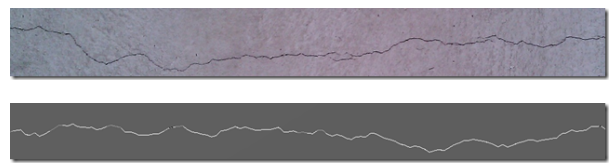

Figure 5: Crack path noise. Complex micro branching observed during crack propagation (top) can be reproduced with fidelity using a noise function (bottom). Similar results by full simulation would require expensive computation.

\subsection{Summary of Required Input}

The input required for a crack simulation is a set of material properties $\left(E, v, \rho, R_{c_{m}}\right)$, an age parameter (i.e. total simulation time), junction density, the parameters that control the crack initiation and propagation $\left(d \sigma_{e}, r_{\text {relax }}, v_{\text {relax }}, R_{c_{v a r}}, l\right)$ and the noise values $A$ and $f$.

The material properties are determined by specifying the 
material observed in the exemplar image. The junction density is computed directly from the exemplar. The age parameter is specified by the user, and the noise values are adjusted manually. Finally, the crack propagation parameters need to be optimized to match the crack pattern. In the next section we propose an automated optimization process, based on a Bayesian approach, to search for the parameters that best match the exemplar image. We use the perceptually motivated visual similarity metric to measure the similarity of the generated fracture patterns to the input image.

\section{Optimization of the Simulation Parameters}

Our goal is to perform the simulation of the previous section, and produce patterns similar to the input described by its statistics. To do this, we need to optimize the values of parameters: $d \sigma_{e}, r_{\text {relax }}, v_{\text {relax }}, R_{c_{\text {var }}}, l$. These are marked "OPT" in the lower part of Table 2. These parameters affect the statistics and thus the visual appearance of the simulated fracture. However, they are hard to set manually since there is no direct way to predict their influence on visual appearance. In addition, finding these parameters requires searching a 5-dimensional space, which is inherently very tedious. We take advantage of the randomness of two parameters of our fracture model, the resistance to fracture and the loading force position, to introduce the use of a Bayesian optimization process, described next.

\subsection{Preprocess}

Material Parameters As noted in the previous section, the values of $\left(E, v, \rho, R_{c_{m}}\right)$ are determined by the material type. These values can be found in standard reference texts [Bow09], and represents an additional set of values given during the creation of the materials in the modeling process. We list the parameters set by the user before the simulation in the upper part of Table 2. All values used in the images of this paper are given in the supplemental material.

Normalization of the Statistics The input statistics $\mathbf{s}_{\text {obs }}$ coming from the images are initially computed with pixelunit length, while the statistics $\mathbf{S}_{\text {sim }}$ from the simulation have the unit length of the virtual world. In order the make the two values comparable, we normalize all our statistics: the areas of the fragment are divided by the total fractured area, the crack lengths by the square root of the total area.

Range of Values for Optimized Parameters We give a valid range for the optimization of each parameter (see Table 3 ) to guide the optimization. To scale with as many materials as possible, the range of possible values is expressed as a function of some known parameters.

\subsection{Optimization of Remaining Parameters}

Since we have a wide range of possible values for the remaining parameters, we need to have a more efficient optimization approach than the brute force algorithm. As the

\begin{tabular}{|cccc|}
\hline Parameter & Min value & Max value & Ref parameter \\
\hline$d \sigma_{e}$ & $0 \%$ & $33 \%$ & $R_{c_{m}}$ \\
$r_{\text {relax }}$ & $0 \%$ & $100 \%$ & $\sqrt{\text { fragment area }}$ \\
$v_{\text {relax }}$ & $0 \%$ & $33 \%$ & $R_{c_{m}}$ \\
$R_{c_{\text {var }}}$ & $0 \%$ & $100 \%$ & $R_{c_{m}}$ \\
$l$ & $0 \%$ & $3000 \%$ & body mass \\
\hline
\end{tabular}

Table 3: Range of valid values for the optimized parameters. Minimum and maximum values are expressed as a percentage of the reference parameter Ref.

distribution of resistance to fracture as well as the force position are random variables, we follow the same approach used in [BZB02] to find our parameters, which is based on an Approximate Bayesian Computation.

We need to find appropriate 5-tuples for the values of the parameters of vector $\mathbf{p}=\left(d \sigma_{e}, r_{\text {relax }}, v_{\text {relax }}, R_{c_{\text {var }}}, l\right)$ which will result in patterns with statistics similar to those measured from the chosen fracture input image. The high level structure of the process is illustrated in Fig. 6.

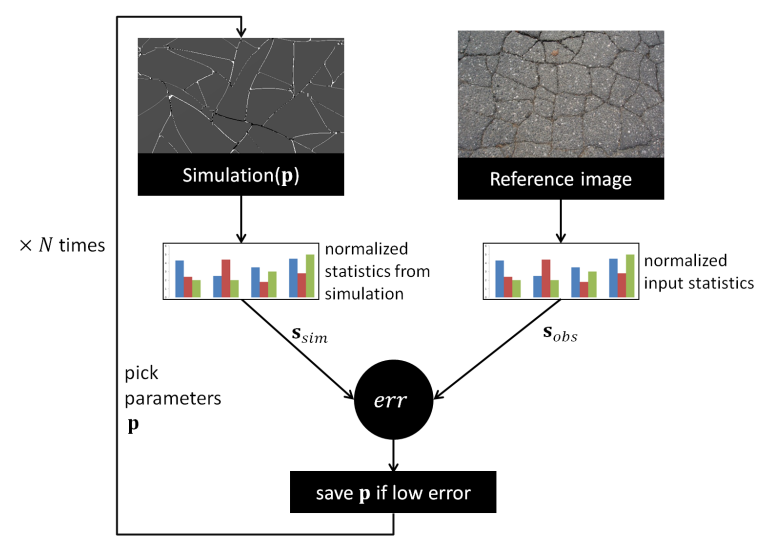

Figure 6: Overview of the optimization process. Parameter vector $\mathbf{p}$ is optimized iteratively. After each simulation, the statistics of the simulation are compared to the statistics of the reference image. The err function is our metric (Eq. 5).

The main principle of the algorithm consists in sampling the five parameters of Table 3 in their respective valid ranges, following a uniform law. At each trial, the distribution of the resistance to fracture is sampled following a normal law. The loading force position is sampled following a uniform law. We run $N$ simulations, each one with a different set of parameters $\mathbf{p}$, and each one generating different output statistics $\mathbf{s}_{\text {sim }}$. After each simulation we compare the fracture input image and the simulated one using the following metric:

$$
\begin{aligned}
\operatorname{err}\left(\mathbf{s}_{\text {obs }}, \mathbf{s}_{\text {sim }}\right)= & w_{\text {frag }} \cdot \operatorname{EMD}\left(\mathbf{s}_{\text {fragobs }}, \mathbf{s}_{\text {fragsim }}\right) \\
+ & w_{\text {crack }} \cdot \operatorname{EMD}\left(\mathbf{s}_{\text {crackobs }}, \mathbf{s}_{\text {cracksim }}\right) \\
+ & w_{\text {junc }} \cdot \operatorname{EMD}\left(\mathbf{s}_{\text {juncobs }}, \mathbf{s}_{\text {juncsim }}\right)
\end{aligned}
$$

where 'frag' stands for fragment area statistics, 'crack' for 
crack length statistics, and 'junc' for junction statistics. Since all the statistics correspond to histograms of values measured in the image or simulation result, we chose to use the Earth Movers Distance [RTG98] (noted EMD in Eq. (5)), which is known to give good results [PW08]. Finally, the $w$ are weighting values that allow to give more importance to some statistics w.r.t. the others. In our examples, we use $w_{\text {frag }}=3, w_{\text {crack }}=1, w_{\text {junc }}=1$. These weights have been selected based on the user study, giving more weight to fragment area statistics than to the other two. For the user study, all weights were set to 1 to generate the synthetic patterns, since the weightings were not known.

At the end of the $N$ simulations, we take the set of parameters with the lowest error computed using our metric. This set of parameters can be then used to generate as many similar patterns as desired, by varying the resistance to fracture and the loading force position. We found that $N=20,000$ produced a good set of parameters for all our exemplars. A rectangular tile geometry, proportioned similar to the exemplar and discretized into 24,000 elements, is used for parameter estimation. Although a single force position is considered during estimation, multiple positions could be easily added to handle more complex patterns.

\section{Interactive Fracture Modeling}

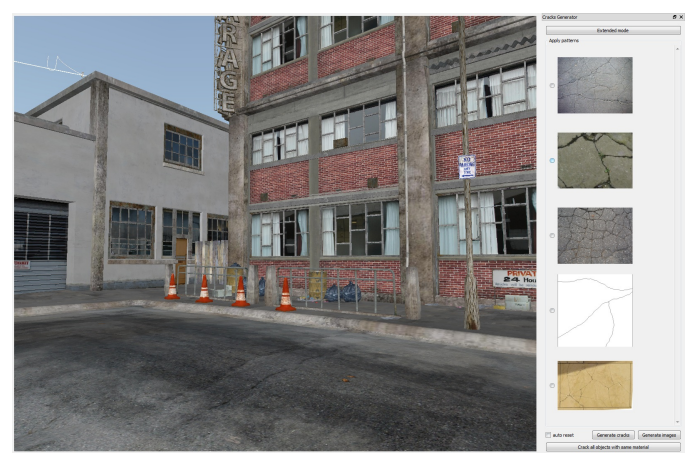

Figure 7: Snapshot of our interface. The user chooses a material from the menu (right); they are subsequently applied to all objects sharing the same material description.

The speed of the underlying simulation allows us to use our results for interactive fracture modelling (Fig. 7). The modeller can choose the parameters associated with an exemplar from a library and apply them to different objects in a scene. The user can then request the system to automatically propagate to all objects with the same material. In the accompanying video we show an example of a modelling session in a city scape, where the fractures are applied to the sidewalk and the road.

\section{Results}

This section shows results of our method applied on different scenarios. Fig. 1 shows several fracture simulations using parameters optimized with our metric. Although the geometries of the simulated objects are very different, the visual features of the input fracture patterns are correctly reproduced. This can also be observed in Fig. 8 and 9.
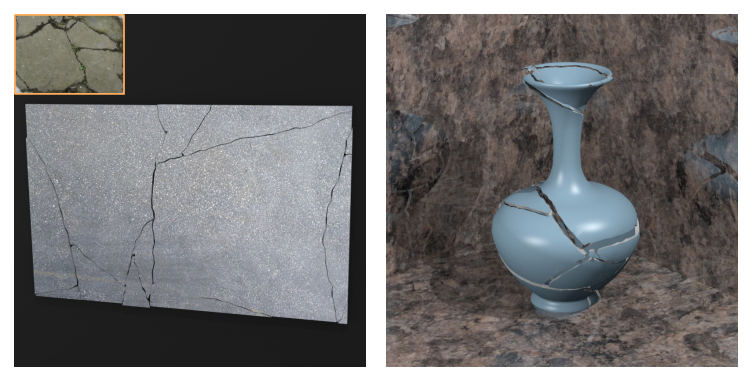

Figure 8: Same pattern applied on different objects, using the exemplar shown in the inset.

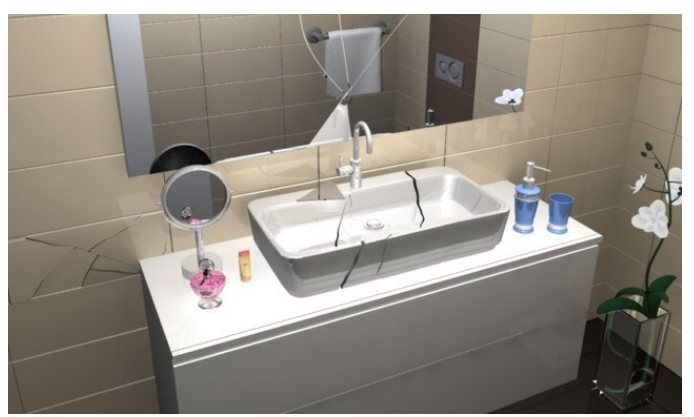

Figure 9: Bathroom scene with several broken objects.

Since we are simulating the interior of the objects, a complete closed surface mesh is generated for each fragment. These fragments can be then moved or removed independently, as shown in the leftmost image of Fig. 1, Fig. 9, as well as the accompanying video and supplemental material.

Once the parameters have been optimized for a specific pattern, we can easily simulate the state of a fractured object at different ages. Fig. 10 shows an example of the state of a road experiencing a loading force at four different ages.

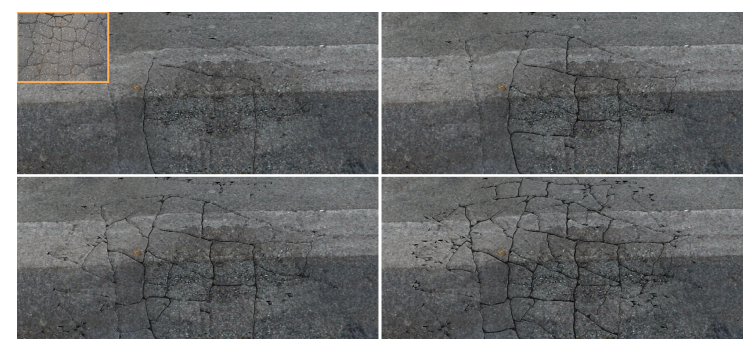

Figure 10: From left to right and top to bottom: Road model showing fracture propagation, simulating the course of time.

Fig. 11 shows an example of an editing session on the urban scene, using our interactive fracture modeller. The editor 

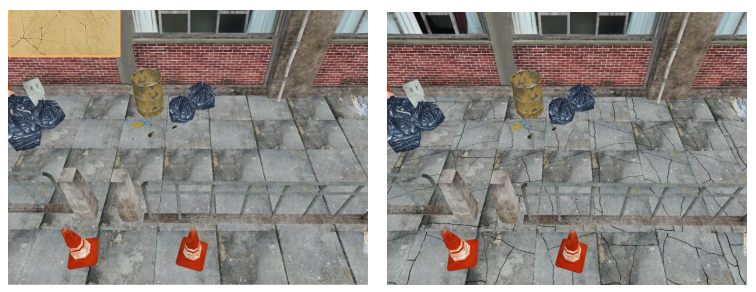

Figure 11: Similar fracture patterns can be automatically generated and propagated from a single input (inset).

provides ways to add external input forces onto the object allowing richer and physically-based fracture patterns. We can also easily propagate a simulated pattern over similar objects, as shown in Fig. 11 right. Please see the accompanying video and supplemental material for further results.

Fig. 12 shows a detailed view on the geometry of the generated fragments for a slab from Fig. 11. The non-flat internal surfaces of cracks are due to our simulation approach. We can thus automatically model fractures that correctly adapt to the $3 \mathrm{D}$ object geometry, its material properties, or external factors such as forces.

The processing of each input exemplar takes between 2 and 5 minutes depending on the number of fragments. The optimization then takes 15 to 25 minutes for 20,000 trials using a body with about $40 \mathrm{~K}$ tetrahedra. Computing each trial involves running the simulation with the current parameters, extracting the statistics of the obtained pattern and computing the difference using our metric based on EMD. Each such trial takes about 60 milliseconds.

The meshes used in our example applications vary from $7 \mathrm{~K}$ tetrahedra for the ground tiles shown in Fig. 11, to 54K tetrahedra for the road model of Fig. 10. The simulation time varies from 16 ms to generate 4-5 cracks and 4-6 fragments on the tile mesh, to $264 \mathrm{~ms}$ for 271 cracks and 71 fragments on the road mesh. The interactive editor runs between 30 and $100 \mathrm{fps}$, depending on the complexity of the scene. The latter framerate is obtained in the urban scene using 100 fracturable bodies along with the rest of the scene. Note however that most of this time is spent in visualizing the models. All timings were obtained in an Intel Core 2 Extreme with 2.3 $\mathrm{GHz}$ and 4GB of RAM, using a nVidia Quadro FX 3700M.

\section{Discussion}

In our approach, we use a physically-based method that generates fracture patterns conforming to the shape of any virtual object (e.g., the sink in Fig. 1). In contrast, it would be hard to apply a procedural method to complex shapes or geometries without additional effort, and patterns would not necessarily respect the true behavior of fracture.

Compared to previous exemplar-based aging and weathering methods that either reused images or exactly replicated observed patterns, we generate fracture patterns using

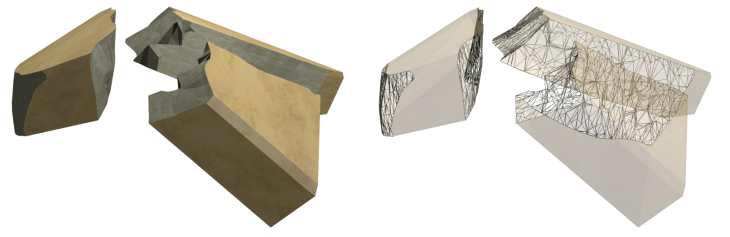

Figure 12: Advantages of the volumetric mesh. The simulation algorithm generates true $3 D$ crack surfaces for realistic rendering. Left: A tile being broken in generates four detailed surface meshes. Right: Visualization of these meshes.

the statistics of a photograph. To do this, we needed to introduce a new metric to compare fracture pattern similarity. We achieved this by a user study, where we first determined that statistics are a valid measure of visual similarity, and then we investigated the relative importance of each category of statistics. Although we covered different categories in our study (e.g., area, edge and junction information), further studies could be performed to evaluate other statistics. Our study introduces a novel way of comparing visual similarities by adding the user perception directly in the loop. In addition, an interesting observation of our perceptual study is that participants seemed to perceive more similarities when the images were optimized by matching the 2D statistical properties (e.g., fragment area or junction angles) rather than 1D (e.g., edge length). This observation could be further explored in future work.

Some limitations of our work concern the volumetric mesh needed to generate fracture patterns. First, the thickness of our meshes needs to be approximately estimated since we do not have any value for material thickness from the example image. Second, the volumetric meshes need to be converted to textured images in an offline process currently. The latter, however, could be easily automated in the future. Volumetric meshes remain an important feature of our approach since they allow the use of continuum mechanics equations during the physically-based simulation step.

\section{Conclusion}

One key question we addressed in this work is whether statistics of fracture patterns are an appropriate measure of visual similarity for fracture patterns. We performed a user study to evaluate user perception for different statistics and thus determine which statistics are correlated to visual similarity. Our study showed that the fracture area and junction statistics have strong effects on visual similarity of fracture patterns. We extended previous physically-based fracture models to allow simulation of a wide range of crack patterns at interactive rates and to be amenable to parameter optimization. We finally proposed a Bayesian optimization method to determine the parameters of the fracture model so it can produce fracture patterns with the same key statistics 
as an exemplar, which are thus visually similar. An important aspect of our optimization approach is the introduction of a fracture similarity metric based on the statistics as evaluated in the user study.

Our results show that our new approach can efficiently reproduce a variety of fracture patterns, allows interactive fracture modeling and thus the processing of large scale environments. We have shown that our approach on matching statistics is well suited for fractures, but we believe it could also be applied to other phenomena. We believe that our method will have significant impact in many applications of digital content creation or architectural and urban design.

\section{Acknowledgments}

This work is partially supported by the Spanish Ministerio de Ciencia e Innovación (grant TIN2010-20590-C0202) and NSF grant NSF-1064412. We acknowledge support from Autodesk (research and software donations), Adobe (research donation) and NVIDIA (hardware donation).

\section{References}

[BHTF07] Bao Z., Hong J.-M., Teran J., Fedkiw R.: Fracturing rigid materials. IEEE Trans. on Vis. and Comp. Graph. 13, 2 (2007), 370-378. 2

[BLR*11] Bosch C., LafFont P.-Y., Rushmeier H., Dorsey J., DretTaKis G.: Image-guided weathering: A new approach applied to flow phenomena. ACM Trans. on Graphics 30 (May 2011), 20:1-20:13. 1, 2

[Bow09] Bower A. F.: Applied Mechanics of Solids. CRC Press, 2009. 7

[BZB02] Beaumont M., Zhang W., Balding D.: Approximate Bayesian computation in population genetics. Genetics 162, 4 (2002), 2025-2035. 7

[CBRY09] Chowdhury A. S., Bhandarkar S. M., RobinSON R. W., YU J. C.: Virtual multi-fracture craniofacial reconstruction using computer vision and graph matching. Computerized Medical Imaging and Graphics 33, 5 (2009), 333-342. 3

[Clo55] Cloos E.: Experimental analysis of fracture patterns. Geological Soc. Of America Bulletin 66, 3 (1955), 241-256. 3

[DGA05] Desbenoit B., Galin E., Akкouche S.: Modeling cracks and fractures. The Visual Computer 21, 8-10 (2005), 717726. 2

[DPH96] Dorsey J., Pedersen H. K., Hanrahan P.: Flow and changes in appearance. In Proc. SIGGRAPH'96 (1996), ACM, pp. 411-420. 2

[FSTF*11] Funkhouser T., Shin H., TOler-Franklin C., Castañeda A. G., BRown B., Dobkin D., Rusinkiewicz S., WEYRICH T.: Learning how to match fresco fragments. $J$. Comput. Cult. Herit. 4 (Nov. 2011), 7:1-7:13. 3

[GC01] Gobron S., Chiba N.: Crack pattern simulation based on 3D surface cellular automata. The Visual Computer 17 (2001), 287-309. 3

[GMD12] Glondu L., Marchal M., Dumont G.: Real-time simulation of brittle fracture using modal analysis. IEEE Trans. on Visualization and Computer Graphics (2012). 2, 4, 5, 6

[Gri21] GRIFFITH A. A.: The phenomena of rupture and flow in solids. Royal Society of London Philosophical Trans. Series A 221 (1921), 163-198. 3
[GTR*06] GU J., TU C.-I., RAmamoorthi R., BelhumeUR P., MATUSIK W., NAYAR S.: Time-varying surface appearance: acquisition, modeling and rendering. ACM Trans. on Graphics 25 (July 2006), 762-771. 2

[HT06] Hsien H.-H., TAI W.-K.: A straightforward and intuitive approach on generation and display of crack-like patterns on 3D objects. In CGI (2006), pp. 554-561. 2

[IO06] IBEN H. N., O'BRIEN J. F.: Generating surface crack patterns. In SIGGRAPH/Eurographics Symp. on Comp. Anim. (2006), pp. 177-185. 1, 2, 4, 5, 6

[LM09] Long J., Mould D.: Dendritic stylization. The Visual Computer 25, 3 (2009), 241-253. 2

[MG04] Müller M., GRoss M.: Interactive virtual materials. In Proc. of Graphics Interface (2004), pp. 239-246. 1, 2

[MG08] MÉrillou S., Ghazanfarpour D.: Technical section: A survey of aging and weathering phenomena in computer graphics. Computers and Graphics 32, 2 (2008), 159-174. 2

[MMDJ01] Müller M., MCMillan L., Dorsey J., JaGnow R.: Real-time simulation of deformation and fracture of stiff materials. In EGWCAS (2001), pp. 113-124. 2

[Mou05] Mould D.: Image-guided fracture. In Proc. of Graphics Interface 2005 (2005), Canadian Human-Computer Communications Society, pp. 219-226. 2

[MST04] MÜller M., SChIRM S., TESChner M.: Interactive blood simulation for virtual surgery based on smoothed particle hydrodynamics. Tech. and Health Care 12 (2004), 25-31. 6

[OBH02] O'Brien J. F., BArgteil A. W., Hodgins J. K.: Graphical modeling and animation of ductile fracture. $A C M$ Trans. on Graphics 21, 3 (2002), 291-294. 1, 2

[OH99] O'BRIEn J. F., Hodgins J. K.: Graphical modeling and animation of brittle fracture. In Proc. SIGGRAPH'99 (1999), ACM, pp. 137-146. 2

[Per02] PERLIN K.: Improving noise. ACM Trans. on Graphics 21 (July 2002), 681-682. 6

[PW08] Pele O., Werman M.: A linear time histogram metric for improved sift matching. In ECCV (2008), pp. 495-508. 8

[RTG98] Rubner Y., Tomasi C., Guibas L. J.: A metric for distributions with applications to image databases. In ICCV (1998), pp. 59-66. 8

[SDF*10] ShIN H., DOUMAS C., Funkhouser T., Rusinkiewicz S., Steiglitz K., Vlachopoulos A., WEYRICH T.: Analyzing fracture patterns in Theran wall paintings. In VAST (Sept. 2010). 2, 3

[TF88] Terzopoulos D., Fleischer K.: Modeling inelastic deformation: viscolelasticity, plasticity, fracture. In Proc. SIGGRAPH'88 (1988), ACM, pp. 269-278. 2

[VPll08] VAlette G., PrÉvost S., LuCAS L., LÉONARD J.: A dynamic model of cracks development based on a 3D discrete shrinkage volume propagation. Comp. Graph. Forum 27, 1 (2008), 47-62. 3

[WTL*06] WANG J., TONG X., Lin S., PAN M., Wang C., BaO H., GuO B., Shum H.-Y.: Appearance manifolds for modeling time-variant appearance of materials. ACM Trans. on Graphics 25, 3 (2006), 754-761. 2

[ZJ10] ZhenG C., JAMES D.: Rigid-body fracture sound with precomputed soundbanks. ACM Trans. on Graphics 29, 4 (2010). 2

[ZTZ05] ZIENkIEWICZ O., TAYlor R., Zhu J.: The Finite Element Method: Its Basis and Fundamentals. ButterworthHeinemann, 2005. 4 